Décadrages Décadrages

cınéma, à travers champs Cinéma, à travers champs

39 | 2018

Jeu vidéo et cinéma

\title{
Taste of Cement (Ziad Kalthoum, 2017), ou le goût d'un engagement en demi-teinte
}

\section{Achilleas Papakonstantis}

\section{(2) OpenEdition}

1 Journals

\section{Édition électronique}

URL : https://journals.openedition.org/decadrages/1366

DOI : 10.4000/decadrages.1366

ISSN : 2297-5977

Éditeur

Association Décadrages

Édition imprimée

Date de publication : 1 décembre 2018

Pagination : 137-152

ISBN : 978-2-9700963-5-1

ISSN : 2235-7823

\section{Référence électronique}

Achilleas Papakonstantis, «Taste of Cement (Ziad Kalthoum, 2017), ou le goût d'un engagement en demi-teinte », Décadrages [En ligne], 39 | 2018, mis en ligne le 05 février 2021, consulté le 04 avril 2022. URL : http://journals.openedition.org/decadrages/1366; DOI : https://doi.org/10.4000/decadrages. 1366 


\title{
Taste of Cement (Ziad Kalthoum, 2017), ou le goût d'un engagement en demi-teinte
}

\begin{abstract}
A Beyrouth, un groupe D'ouvriers venus de Syrie travaillent à la reconstruction d'un gratte-ciel; le soir, soumis au couvre-feu imposé par les autorités libanaises, il leur est interdit de quitter le site de construction et de passer leur temps libre en ville. C'est alors dans un dortoir souterrain au pied du site qu'ils s'abritent pour la nuit. Célébré par la quasi-totalité de la critique francophone comme un chef-d'œuvre du cinéma documentaire, le deuxième film de Ziad Kalthoum, Taste of Cement, a également rencontré le succès dans l'arc lémanique. Sélectionné en compétition internationale à l'édition 2017 des Visions du Réel, il a non seulement suscité l'engouement de la part du public mais a également remporté le Sesterce d'Or du festival. Ce n'était que le début; récompensé en automne aux festivals de Camden en Angleterre et d'Adélaïde en Australie, le film a bénéficié d'une sortie commerciale en France au début de l'année 20I8'. Distribué en Suisse par Cinélibre (association des ciné-clubs et des cinémas à but non lucratif), Taste of Cement a été projeté au Cinéma Oblò à Lausanne en janvier et février; la première de ces projections, le ir janvier, a eu lieu en présence de Kalthoum, alors de retour en Suisse romande pour présenter son film et discuter avec le public du rôle des cinéastes syriens face à la réalité de la guerre qui ravage leur pays ${ }^{2}$. Or, le jeune réalisateur (né en 198 à à Homs, centre industriel et économique de la Syrie qui fut un des épicentres des affrontements dès le début de la guerre en $20{ }^{3}{ }^{3}$ ) était déjà connu du public suisse puisque son premier long métrage, Le Sergent immortel,
\end{abstract}

1 En mai 2018, l'exploitation commerciale du film en Allemagne était alors en cours.

2 La discussion fut animée par Nicolas Appelt, actuellement assistant pour le Master Moyen-Orient du Global Studies Institut (Université de Genève) et spécialiste du cinéma et des séries télévisées syriens (voir son article «Présence du cinéma syrien», Décadrages, $n^{\circ} 3$, automne 20I5, Pp. I37-I4I). Par ailleurs, Sandy Fernandez, membre du comité de gestion du Cinéma Oblò, a facilité notre accès aux films de Kalthoum ainsi qu'à leur réception critique; qu'elle soit ici remerciée pour son aide précieuse.

3 Homs est la troisième ville la plus peuplée du pays, située au bord du fleuve de l'Oronte que les indigènes appellent - ironie de l'histoire - le «fleuve rebelle». De fait, il s'agissait de l'un des bastions du soulèvement contre le régime d'Assad, d'où son surnom «Capitale de la Révolution». Comme le soulignait l'anthropologue Thierry Boissière en 2012 déjà, " $[\mathrm{d}] \mathrm{e}$ toutes les grandes villes syriennes, Homs est celle qui a pour l'instant payé le prix le plus élevé pour le rôle qu'elle joue dans la Révolution syrienne. L'acharnement dont le régime de Damas fait preuve à son encontre ne peut cependant se comprendre complètement sans prendre en compte la place singulière que la ville a occupée et occupe encore dans la région et le pays». Nous renvoyons le lecteur à son article très instructif sur la guerre civile en Syrie comme une guerre des classes: Thierry Boissière, «Homs, capitale de la révolution, carrefour alaouite», Mediapart, II mai 2012, en ligne: https://blogs.mediapart.fr/edition /les-invites-de-mediapart/article/ıIo5I2 /homs-capitale-de-la-revolution-carre four-alaoui [consulté le 2ı mai 20ı8]). 
4 Rappelons ici que, d'après François Jost, une image devient subjective «lorsque se marque dans le signifiant la matérialité d'un corps» identifié comme le sujet du regard. L'auteur propose par la suite une liste non exhaustive de cinq critères permettant d'identifier l'emploi d'une ocularisation interne primaire; deux parmi eux - «[1]a représentation d'une partie d'un corps en premier plan, supposant l'ancrage dans un regard» et «l'ombre du photographe»- sont présents dans le cas du Sergent immortel. Voir François Jost, L'OEil caméra: entre film et roman, Lyon, PUL, I987, pp. 24-26. fut projeté au Festival International du Film de Locarno en 20I4 (dans la section Signs of Life) et au Festival International de Films de Fribourg (FIFF) en 20I5. Revenant sur l'histoire personnelle de Kalthoum qui, au début de la guerre, s'est trouvé partagé entre son service militaire et son poste d'assistant réalisateur sur le tournage d'Une échelle pour Damas (Mohammad Malas, Syrie/Liban/Qatar, 2013), ce film témoignait déjà du penchant du réalisateur pour le récit autobiographique et la mise en œuvre de stratégies énonciatives subjectivantes, éléments qui - comme nous essaierons de le montrer dans cet article - jouent également un rôle structurant dans la construction narrative de Taste of Cement.

\section{L'œil surplombant de la machine}

Dans une succession de vues aériennes qui défilent pendant le générique de début et par le biais de mouvements fluides et continus, la caméra balaie un paysage rocheux à matrice argileuse, ingrédient nécessaire à la fabrication du ciment ( fig. I-2). L'appareil de prise de vues gagne ensuite en hauteur et se dirige vers le ciel de Beyrouth dont les bâtiments deviennent visibles derrière les collines au gré de ce mouvement ascendant. Ainsi, Taste of Cement s'amorce par un enchaînement de plans qui laisse supposer une rupture - quoique non radicale - sur le plan énonciatif par rapport au film précédent du cinéaste. De fait, l'emploi systématique et exclusif de la caméra subjective dans Le Sergent immortel ne contribue pas seulement à l'exhibition frontale des modalités d'expression filmique, mais également à l'ancrage de l'énonciation par rapport à un sujet humain. L'acte de filmer est au centre même du récit; Kalthoum parcourt les rues de Damas caméra à la main, inscrivant ses mouvements, ses hésitations et le rythme même de ses déplacements au centre de la facture de son film. Filmer à la première personne lui donne la possibilité de répondre à l'intuition de l'instant et d'immortaliser les contingences de la vie en période de guerre. Le cinéaste reste toujours hors champ (avec l'exception ponctuelle mais notable de ses jambes et de ses pieds) alors que la subjectivité de l'image est affirmée à la fois par son ombre omniprésente et par les regards caméra de ses interlocuteurs ${ }^{4}$.

Dans la séquence d'ouverture de Taste of Cement, l'ombre du «filmeur» fait également irruption dans le champ, sauf qu'il ne s'agit plus d'un être humain mais d'un drone. Si l'énonciation reste marquée (ce qui n'est guère surprenant pour un film documentaire), elle est cette fois mé- 
canique, voire impersonnelle. Certes, d'après Christian Metz, ce constat serait valable pour toute image enregistrée par une caméra ${ }^{5}$; or, l'intégration de l'appareil de prise de vues dans un dispositif semi-autonome, manipulé à distance, ne peut qu'exacerber la tension induite par l'absence d'un sujet humain. De la sorte, parler ici d'images subjectives serait pour le moins paradoxal. Les montagnes, les rues et les bâtiments de Beyrouth sont filmés depuis le point de vue d'un surhumain ou d'un outre-humain capable de voler, d'un oil machinique qui plane au-dessus de la population libanaise. Aussi trivial qu'il puisse paraître à première vue, l'emploi du drone instaure dès les premières minutes un régime énonciatif qui, d'après notre analyse, préfigure le discours politique construit et véhiculé par l'ensemble du film. Dispositif exemplaire du nouveau paradigme de la guerre qui émerge au $\mathrm{XxI}^{\mathrm{e}}$ siècle, le drone ne fait qu'intensifier un vieux processus stratégique de dépolitisation des conflits armés au profit
5 Voir Christian Metz, L’Enonciation impersonnelle ou le site du film, Paris, Méridiens Klinksieck, I99I.

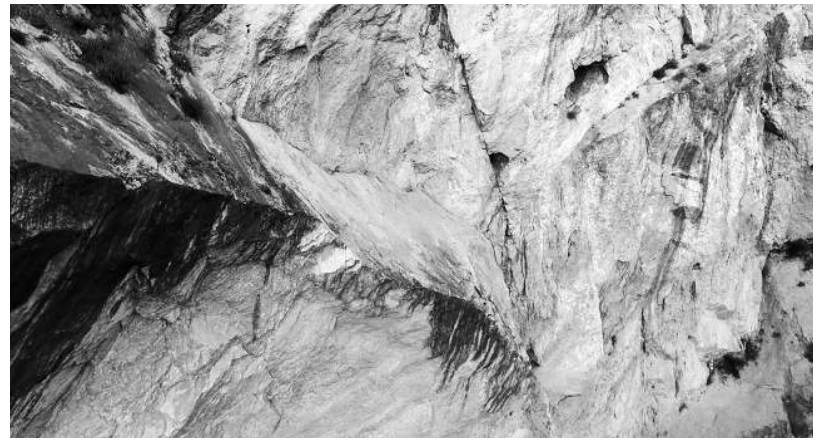

1

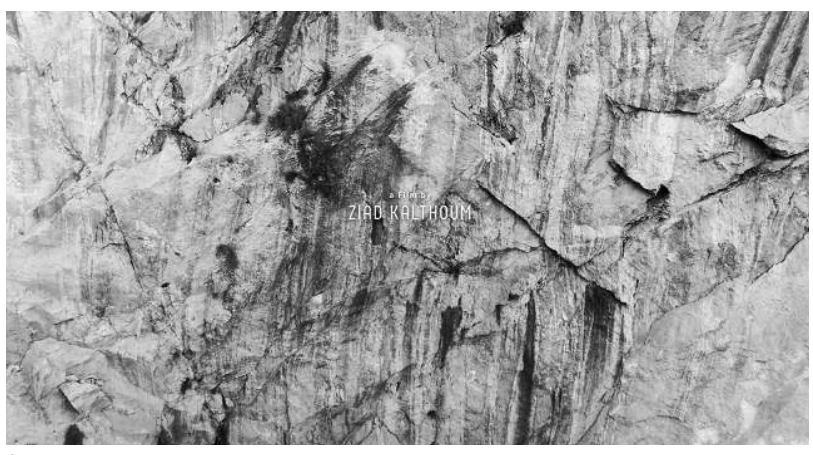


6 Pour une analyse plus détaillée des drones dans le cadre d'une archéologie des dispositifs de vision, nous renvoyons le lecteur au dossier «Drones, cartographie et images automatisées », dirigé par Claus Gunti dans Décadrages, $\mathrm{n}^{\circ}$ 26-27, printemps 20I4; pour une réflexion ciblée sur les enjeux éthiques de l'emploi militaire de drones, voir plus précisément l'article de Marc-André Weber dans le même dossier, «La guerre des irresponsables» (pp. 98-ıго). Enfin, l'ouvrage philosophique de Grégoire Chamayou, Théorie du drone (Paris, La Fabrique, 2013) et le livre de Lisa Parks et Caren Kaplan, Life in the Age of Drone Warfare (Durham, Duke University Press, 20I7) constituent à notre avis des lectures incontournables sur ces questions.

7 Marc-André Weber, op. cit., p. Io4. Et un peu plus loin, «les militaires eux aussi occupent la posture du spectateur. Un opérateur de drone ne passet-il pas l'essentiel de son temps à regarder un écran?» (p. Io6). Précisons pourtant qu'une assimilation complète entre la posture de l'opérateur de drone et celle de spectateur de cinéma serait erronée, ne serait-ce que parce que le premier peut (et même doit) directement influer sur la représentation; à cet égard, le rapprochement avec la posture vidéoludique nous paraît plus exact.

8 Paul Virilio, Guerre et cinéma. Logistique de la perception, Paris, Editions de l'Etoile/Cahiers du cinéma, I984. de leur aspect spectaculaire ${ }^{6}$. On le sait, l'émergence de la guerre automatisée a permis aux combattants (se situant, bien entendu, du côté des «riches» sur le continuum technico-financier de notre planète) d'être physiquement absents du champ de bataille, transmués désormais en acteurs-spectateurs. Comme le remarque Marc-André Weber en se mettant à la place d'un auteur des bombardements par drone interposé, «[] es morts et les blessés qu'il cause sont des images floues sur un écran ${ }^{7}$; il semblerait alors que, même pour ceux qui la mènent, la guerre s'est définitivement transformée en spectacle.

Reprocher à Taste of Cement un effet de spectacularisation de la guerre en Syrie (et plus spécifiquement de la situation des réfugiés ayant réussi à traverser la frontière libanaise) sur la seule base de l'emploi d'images enregistrées par un drone constituerait à n'en pas douter une assertion hâtive - ne serait-ce qu'à cause du fait que, au-delà des trois premières minutes, on ne trouvera pas d'autres plans tournés avec le même dispositif de filmage. Notre analyse se concentrera donc sur un certain nombre de stratégies formelles et narratives qui structurent l'ensemble du film et accordent à l'instance énonciative (et par extension au spectateur) la place d'un observateur désengagé. Quant à la séquence inaugurale, nous nous contenterons ici de formuler deux remarques supplémentaires.

En premier lieu, l'emploi d'images enregistrées par un drone au sein d'un film documentaire témoigne de la généralisation des usages de ce type de dispositif tout en participant activement à sa normalisation. Ainsi, Taste of Cement s'inscrit dans un ensemble, de plus en plus large, de films récents qui accoutument le public à cette nouvelle technologie; or, faut-il encore le rappeler, le drone est un outil destiné non seulement aux cinéastes, mais surtout et avant tout aux services de surveillance et aux militaires, endossant régulièrement la fonction d'arme de combat. Certes, dans la deuxième moitié des années 20ı,, la «domestication» des drones ne s'effectue pas seulement à travers le cinéma, mais également par d'autres types d'usages, notamment ludiques ou pédagogiques, accessibles au plus grand nombre. Il existe toutefois un lien historique particulièrement étroit entre le développement des technologies de prise de vues et celles de l'armement militaire, analysé dans ses multiples facettes par Paul Virilio dans les années I980 ${ }^{8}$. De nos jours, à l'ère de l'hyper-connectivité, de la prolifération des écrans et du «régime média- 
tique de l'alerte ${ }^{9}$ - manifesté notamment à travers un état global de surveillance-, le rapport stratégique entre l'industrie du spectacle et celle de la guerre se poursuit principalement en cultivant et en exploitant «une série de fantasmes relatifs à la possibilité d'une maîtrise étendue du voir et du savoir ${ }^{10}$, voire un désir généralisé d'omniperception et d'ubiquité, nourri en outre par la paranoïa anti-terroriste qui a envahi le monde occidental. Il est essentiel à cet égard de relever que l'emploi du drone dans la séquence liminaire de Taste of Cement ne produit pas seulement des effets rythmiques et stylistiques ${ }^{11}$, mais présente également des implications narratives: à la différence d'une immersion par focalisation interne stricte telle que le spectateur l'éprouvait dans Le Sergent immortel, nous nous trouvons ici dès le début face à une représentation ubiquitaire, déployée par une instance narrative à distance (cognitive, émotionnelle, physique) par rapport aux personnes filmées, située significativement au-dessus d'elles. La voix over qui intervient dès la deuxième séquence du film (nous y reviendrons) corrobore cette hypothèse d'un narrateur/ énonciateur en surplomb.

En deuxième lieu, il est assez ironique que Kalthoum ait recours à cette technologie pour filmer la ville de Beyrouth; trente ans plus tôt, dans son ouvrage de référence susmentionné, Virilio décrit en ces termes le vol d'un drone au-dessus de la capitale du Liban: «œil de Tsahal équipé de caméras TV et de systèmes d'imagerie thermique survolant Beyrouth assiégée, au ras des toits des immeubles, au-dessus des quartiers palestiniens les plus exposés, affichant sur les consoles de visualisations à plus de cent kilomètres de là, pour les analystes israéliens, l'image des déplacements de populations, le graphique thermique des véhicules palestiniens » ${ }^{12}$. Il s'agissait en l'occurrence du premier déploiement d'un drone dans une situation de guerre, lors de l'opération «Paix en Galilée», inscrite dans l'offensive dite «préventive» lancée par Israël dans le sol du Liban en été I982. Le recours à la même technologie pour enregistrer des images de la même ville dans un film qui aborde frontalement les derniers épisodes dans la longue histoire des conflits au Proche-Orient ne peut que soulever des enjeux politiques, idéologiques, voire éthiques. Nous nous efforcerons de prendre précisément la mesure de la manière dont le film de Kalthoum se positionne face à cet héritage historique extrêmement lourd.
9 Voir le sous-chapitre «Régimes attentionnels» dans Yves Citton, Pour une écologie de l'attention, Paris, Seuil, 2014, pp. 65-7I.

10 Mireille Berton, «Technologies numériques et fantasmes panoptiques dans les séries télévisées contemporaines", Décadrages, $\mathrm{n}^{\circ}$ 32-33, printemps $20 \mathrm{r} 6$, p. 38 .

11 Les travellings contemplatifs et les mouvements lents de la caméra dans cette séquence sont indicatifs de l'esthétique soignée de l'ensemble du film (composé majoritairement de plans fixes et de cadrages extrêmement travaillés), se situant aux antipodes du Sergent immortel, revendiquant pour sa part l'héritage du cinéma «direct», exemplifié par les mouvements brusques de la caméra portée.

12 Paul Virilio, op. cit., p. I47. 


\section{Jeux de guerre}

Le rôle introductif accordé aux vues aériennes témoigne de l'importance du statut de ces images dans l'organisation discursive de Taste of Cement. Une fois la caméra dirigée vers les gratte-ciel de Beyrouth, on entre dans le récit à proprement parler; ce dernier, à l'image du mouvement ascendant qui clôt la séquence d'ouverture et de la contre-plongée marquée du plan suivant (qui, filmé depuis le sol avec une grande profondeur de champ, exagère la perspective et, par conséquent, la perception de la hauteur des deux bâtiments cadrés), se déclinera tout au long du film sur un axe vertical. (fig.3-4) En effet, le film suit la routine quotidienne d'un groupe d'ouvriers syriens qui se sont réfugiés au Liban juste après le début de la guerre. Le jour, ils construisent un gratte-ciel au cœur de Beyrouth; le soir, à partir de dix-neuf heures et tout au long de la nuit, jusqu'à sept heures le matin suivant, un couvre-feu les contraint à se retirer sous l'édifice de ciment que la main-d'œuvre qu'ils constituent met en place jour après jour. Le récit est ainsi rythmé par leur va-et-vient, de haut en bas et inversement, érigeant les motifs de l'ascension et de la descente en principes à la fois narratifs et esthétiques.

Or, le discours de Taste of Cement se construit à travers des jeux de symétrie qui vont bien au-delà des déplacements dédoublés des bâtisseurs (en haut/en bas). Force est de constater que le processus circulaire que l'on pourrait résumer par la formule «construction-destruction(re)construction» constitue la colonne vertébrale du récit: alors que la guerre détruit leurs propres maisons et celles de leurs familles en Syrie, les ouvriers au centre du film sont en train de construire un immeuble
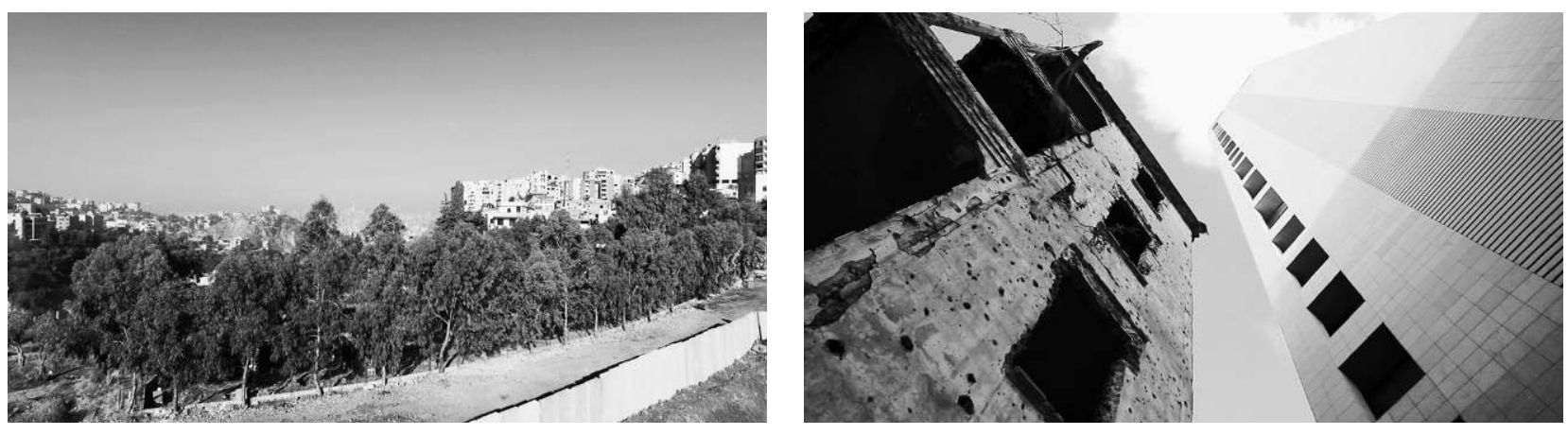
dans un pays étranger qui, dévasté lui aussi par des années de conflits, cherche toujours à se rétablir - sous la menace persistante d'une nouvelle guerre qui pourrait à tout moment éclater et réalimenter ce cercle vicieux. Le plan en contre-plongée des deux immeubles, évoqué plus haut, visualise de manière éloquente cette succession alternée entre les forces de création et de destruction: un panoramique horizontal à I $80^{\circ}$ relie les deux bâtiments, l'un délabré ou en construction (dans tous les cas clairement inhabité), l'autre en état de fonctionnement, un gratte-ciel au style standardisé qui monte jusqu'à se confondre avec les nuages dans le ciel. La caméra s'arrête significativement entre les deux, paralysée face à la confusion temporelle: lequel des deux symbolise l'avenir pour la ville de Beyrouth et lequel représente le présent furtif? Le bâtiment inhabité constitue-t-il un chantier en attente de sa construction? Ou est-ce plutôt le gratte-ciel qui attend le retour inévitable de la guerre? L'incertitude dégagée par la situation géopolitique extrêmement critique de la région attribue à ce jeu de miroir une ambiguïté irréductible. Plus loin dans le film, en ouverture de la troisième et dernière partie, la mise en relation des travaux de reconstruction de la capitale libanaise avec la machine de guerre qui détruit les villes syriennes se matérialise via une séquence en montage parallèle fortement teintée de recherche esthétique: une série des plans montrant les ouvriers en train de travailler, leurs gestes saisis sous des angles de prise de vue multiples, se trouve entrecoupée de plans «subjectifs» enregistrés depuis l'intérieur d'un char d'assaut (avec son canon visible en premier plan), alors que ce dernier parcourt Damas ruinée en tirant sur des bâtiments déjà éventrés (fig.5-6). Le rythme de
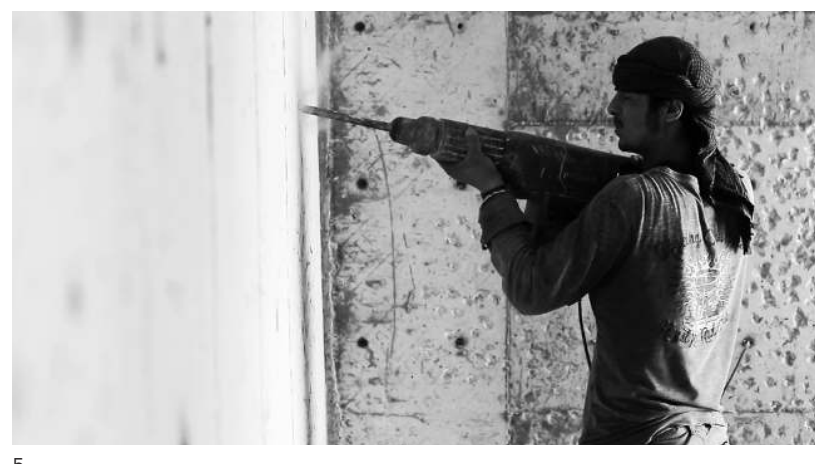

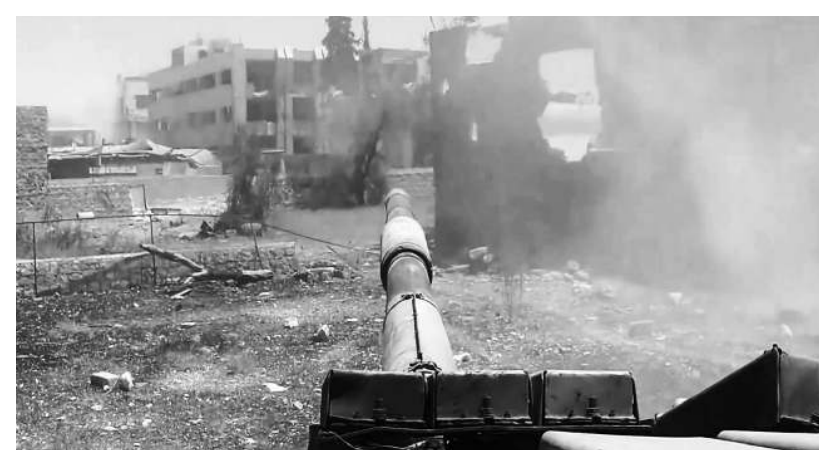

6 
13 Ce constat ainsi que l'analyse qui s'ensuit ne prétendent en aucun cas épuiser la problématique de l'incorporation des conditions de vie liées à la réalité de la guerre et de l'exil, constituant assurément une des thématiques centrales de Taste of Cement. Il ne serait pas exagéré de postuler que le film de Kalthoum érige le corps au rang de medium politique fondamental, en tant que site d'absorption des expériences historico-politiques et de construction contingente de soi. Il s'agit d'ailleurs d'un point exploré sur un ton onirico-poétique par le narrateur over dans la première partie du film: en racontant le souvenir des retrouvailles avec son père après son long séjour au Liban (où ce dernier était, lui aussi, parti travailler en tant que bâtisseur après la fin de la guerre civile libanaise au début des années I990), le narrateur insiste sur la manière dont l'expérience d'immigration de son père fut gravée sur la paume de ses mains: «Whenever he came back for a visit, I'd stare into his hands for hours, memorizing the lines, street by street, so cracked and rough. My father's palm was the city of Beirut» $\left[9^{\mathrm{e}} \mathrm{min}\right]$.

14 Dans un article publié dans The Guardian deux semaines avant l'entrée en fonction officielle de l'administration Trump, Medea Benjamin, auteure du livre Drone Warfare: Killing by Remote Control (Londres, Verso, 2013), rapportait qu'en 20ı6, les Etats-Unis avaient largué 26 I7 I bombes dans l'ensemble de leurs opérations militaires dans le monde, à savoir l'équivalent de trois bombes par heure (Medea Benjamin, "America dropped 26,I7 I bombs in 20I6. What a bloody end to Obama's reign», The Guardian, 9 janvier 20I7, en ligne: www. theguardian.com/commentisfree/20I7/

l'alternance s'accélère progressivement, les ambiances sonores s'entremêlent dans une harmonie déconcertante et la juxtaposition des deux scènes acquiert le caractère d'une expérimentation formelle (avec des effets esthétiques plutôt que rhétoriques ou conceptuels).

Néanmoins, par un travail méticuleux sur le son, le montage et la composition des cadres, Taste of Cement articule habilement ces deux ensembles symétriques - d'un côté la montée (au ciel) et la descente (aux enfers), de l'autre les forces de création et de destruction - au sein d'un discours métaphorique qui explicite l'absurdité de la situation de ces ouvriers syriens et, d'un point de vue plus macroscopique, l'inhumanité de la guerre, voire de toute guerre. Ainsi, la représentation crée de manière systématique l'impression que les bâtisseurs alternent, au fil de leur parcours quotidien, entre la posture physique des «auteurs» des bombardements aériens et celle de leurs victimes ${ }^{13}$. Le jour, ils montent au sommet du chantier, surplombant Beyrouth et ses bâtiments, ses rues remplies de voitures, ses milliers d'habitants. L'analogie entre ces hommes en train de reconstruire une ville et les entités - humaines et non humaines - qui détruisent jour après jour, depuis le ciel, des surfaces habitées quelque part dans le monde ${ }^{14}$ passe d'abord par le cadrage. A la quatorzième minute, on trouve un plan emblématique de cette stratégie rhétorique: la caméra est placée sur le bras d'une des grues mobiles du chantier; ce dernier, en pleine rotation, est partiellement visible en premier plan, alors qu'une autoroute et plusieurs bâtiments occupent l'arrière-plan de cette vue en plongée, filmée en grande profondeur de champ (fig.7). La composition du cadre ainsi que sa mobilité «mécanique» établissent une association visuelle immanquable entre le bras de la grue et un canon qui viserait de manière menaçante l'espace urbain. Ce mode de figuration de la technologie de construction infléchit la manière dont le spectateur perçoit les nombreux plans du film qui montrent les ouvriers de dos

jan/og/america-dropped-26I7I-bombs20ı6-obama-legacy [consulté le 20 mai 20I8]). D’après le Réseau syrien des droits de l'homme (SNHR), ONG fondée en 20II, le régime d'Assad a largué 68334 bombes en Syrie entre juillet 2012 et décembre 2017 , provoquant la mort immédiate de plus de dix mille personnes (Gareth Browne, «Assad drops almost 70,000 barrel bombs on Syria, study finds", The National, 3 janvier 20r8, en ligne: www.thenational.ae/ world/mena/assad-drops-almost-7oooo-barrel-bombs-on-syria-study-finds -ı.692570 [consulté le 20 mai 20I8]). 
sur le chantier, leurs corps dominant la ville, ou ceux qui les cadrent en amorce, alors qu'ils sont en train de manipuler les grues, compositions qui favorisent les allusions aux configurations visuelles des jeux vidéo qu'on qualifie de third-person shooter («tir à la troisième personne»), anticipant en outre les vues subjectives depuis l'intérieur d'un tank, déjà évoquées, qui n’arriveront que vers la fin du film ( fig. 8). La métaphore est par ailleurs renforcée par la bande sonore: la voix over propose un rapprochement, plus ou moins explicite, entre le bruit étourdissant des machines qui œuvrent sur le chantier et celui des bombardements et des mitraillettes : «la première frappe de fer étouffe toute peur, le bruit du martèlement vous soulage» $\left[\mathrm{IO}^{\mathrm{e}} \mathrm{min}\right]^{15}$.

Au coucher du soleil, les ouvriers commencent leur descente, traversant la distance physique qui sépare les deux pôles extrêmes de ce jeu de rôles morbide. Ils se dirigent vers une cavité souterraine, leur

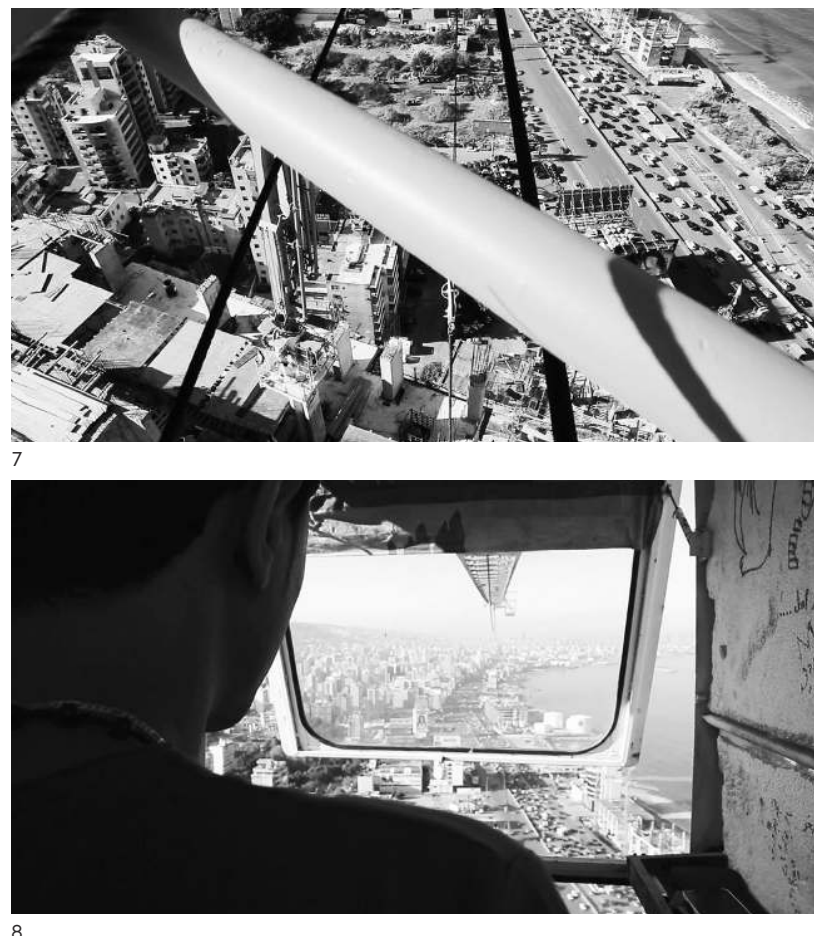

15 «The first iron strike stifles all fear. The sound of pounding relieves you» (notre traduction ici et dans la suite de l'article se base sur les sous-titres anglais de la version numérique du film distribuée par Cinélibre). Notons par ailleurs que le bruit mécanique produit par les différents engins de chantier domine la bande sonore pendant les premiers plans du film, enregistrés - rappelons-nous par un drone. 
16 Suivant la terminologie de Jost, on parle d'une ocularisation interne secondaire «lorsque la subjectivité d'une image est construite par le montage, les raccords (comme dans le champcontrechamp) ou par le verbal (cas d'une accroche dialoguée), en bref, par une contextualisation » (François Jost, op.cit., p. 27). marche imprégnée d'un stoïcisme semblable à celui des habitants d'une ville habitués à prendre les chemins des abris lorsque les sirènes retentissent. Une fois sous terre, le film s'appuie sur une pluralité de stratégies formelles pour suggérer une analogie entre la réalité physique des bâtisseurs du film et celle des victimes des bombardements. Alors que la caméra explore les différents coins de cette «ville» clandestine, improvisée par les ouvriers et négligée par la lumière du soleil, la bande sonore reproduit des reportages journalistiques sur le destin tragique des civils tués ou piégés par les bombardements en Syrie, ainsi que sur le dur travail des sauveteurs qui cherchent des survivants dans les ruines. Des images de débris et de bâtiments écroulés à Damas et à Alep, diffusées à la télévision ou sur internet, interviennent ponctuellement, en ocularisation interne secondaire ${ }^{16}$, contemplées en silence par les bâtisseurs juste avant d'aller se coucher, sur les petits écrans qu'ils ont apportés avec eux ou sur leurs téléphones mobiles. Et après trente minutes de film un plan révélateur de cette analogie apparaît: un plan fixe cadre de profil le corps allongé de l'un des ouvriers, endormi sur le dos, tandis que l'image de quatre bombes, lancées par un hélicoptère, s'inscrit en surimpression; leur parcours est suivi par la caméra à travers un mouvement descendant rapide (fig. 9). Au moment où les bombes se superposent exactement, au sein du cadre, au corps du jeune homme endormi, l'explosion retentit. La voix over revient, quelques secondes plus tard, pour raconter encore un souvenir du narrateur: or, il n'est pas anodin que ce dernier choisisse ce moment précis pour partager avec le spectateur son expérience personnelle en tant que victime des bombardements, piégé dans les ruines

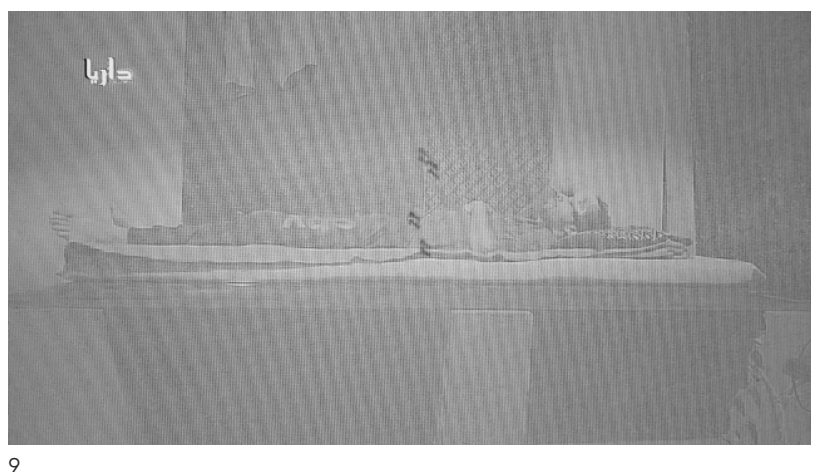


de sa maison et sauvé in extremis. En revenant ensuite au présent et à sa situation d'exilé et d'ouvrier au Liban, son récit rend la comparaison on ne peut plus explicite: «Je me suis bientôt retrouvé enterré dans un autre trou. Ils m’ont dit: 〈pas de bombardements ici〉. Mais je suis toujours entouré de ciment. Je ne peux pas m’échapper.» [32 $\mathrm{min}]$.

La mise en place de cet ensemble d'analogies et de métaphores, telles que nous les avons exposées jusqu'ici, constitue sans aucun doute l'un des points forts de Taste of Cement, déterminant à la fois sa structure narrative et son organisation formelle. Cependant, notre étude serait lacunaire si elle faisait l'économie de l'analyse des effets de sens produits par l'articulation de ces figures stylistiques et rhétoriques - étape nécessaire pour la reconstitution et, éventuellement, l'évaluation du discours construit par le film. A cet égard, les plans sur lesquels s'achève Taste of Cement semblent offrir au spectateur les clés nécessaires à son interprétation. Dans le premier de ces plans, l'ascenseur utilisé par les ouvriers pour leurs déplacements quotidiens sur le chantier est montré en train de descendre; or, la durée longue de ce plan fixe donne le temps nécessaire au spectateur pour s'apercevoir que l'image qu'il regarde n'est que le reflet inversé du chantier, formé sur la surface d’un étang: nos repères directionnels se trouvent brouillés, l'ascenseur est en réalité en train de monter (fig. Io). Les deux plans suivants sont enregistrés depuis l'intérieur d'une bétonnière alors que cette dernière traverse Beyrouth en transportant le ciment utilisé dans les différents chantiers de la ville. La caméra étant alors placée sur une surface en pleine rotation circulaire avec son regard dirigé vers la rue à l'extérieur, les véhicules et les

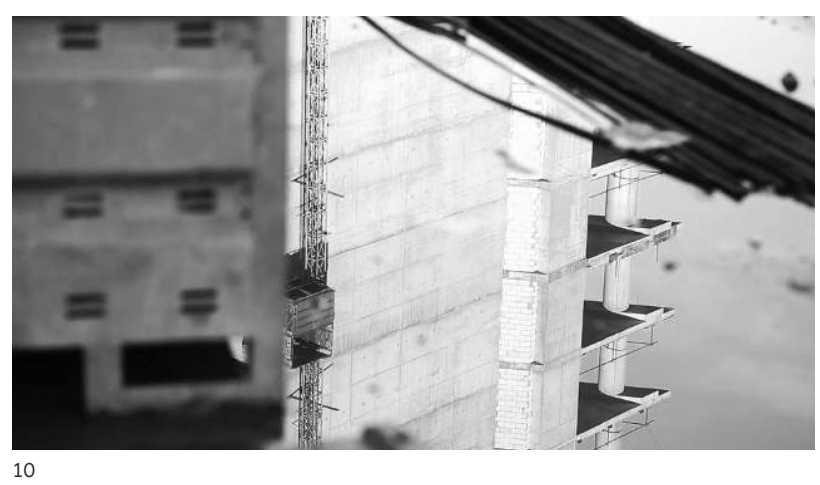




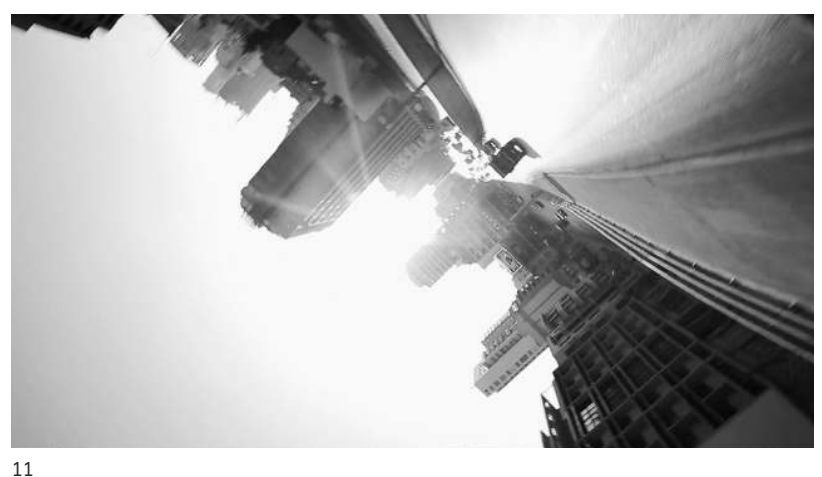

11

bâtiments visibles dans le champ semblent tourner en rond; tout au long de ce plan (dont la durée dépasse les quatre minutes), la répartition entre le haut et le bas perd son importance tandis que tous les éléments du cadre se trouvent engagés dans un échange perpétuel de positions (fig. II). Considérés à la lumière de la verticalité - qui constituerait ainsi non seulement le motif structurant du récit de Taste of Cement, mais également le centre du réseau connotatif et discursif du film--, ces plans acquièrent une importance qui va bien au-delà de leur intérêt plastique: en communiquant les idées de réversibilité et d'instabilité, voire même d'incertitude et d'incompréhension, ils résument en quelques minutes le positionnement politique du film face à la réalité qu'il vient d'exposer, à savoir la situation des réfugiés syriens au Liban et, plus globalement, la guerre actuelle en Syrie.

\section{Normes festivalières et limites de la portée politique}

On l'aura compris, en offrant un témoignage des conditions de vie inhumaines d'un groupe d'ouvriers à Beyrouth, le film de Kalthoum dénonce ouvertement la montée préoccupante du racisme anti-syrien au Liban. Notons que ce pays a accueilli plus d'un million de réfugiés en six ans - bien plus que l’intégralité de l'Union européenne -, de sorte qu'aujourd'hui un habitant sur six est Syrien. Parallèlement à la mise en place de plusieurs programmes de volontariat et d'ONG, consacrés au soutien de personnes échappant à la guerre (à l'instar de l'organisation «Beyond» qui prend en charge la scolarité des enfants syriens), une forme d'exaspé- 


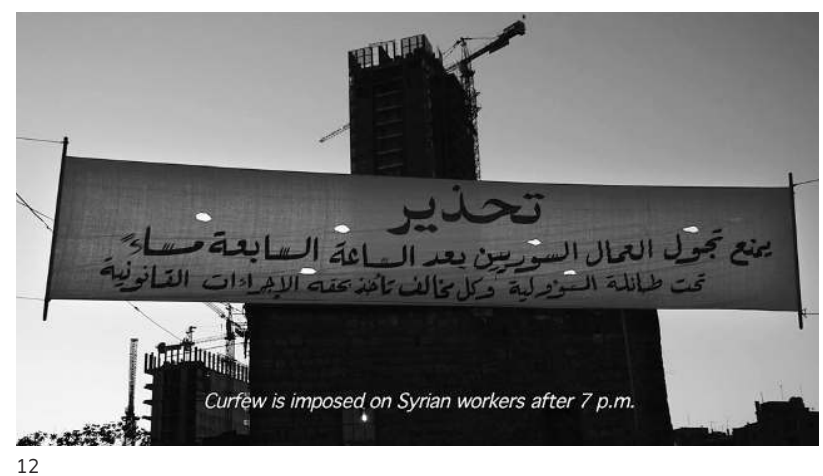

ration contre la présence accrue des réfugiés sur le territoire libanais n’a pas tardé à se manifester. Sur les murs de la capitale, les graffitis insultant les Syriens se multiplient, comme d'ailleurs les manifestations qui mobilisent des slogans accusant l'immigration de masse de concurrence déloyale sur le marché de l'emploi ${ }^{17}$. Le couvre-feu imposé aux bâtisseurs du film par leurs patrons libanais n'est donc qu'un exemple parmi d'autres d'une discrimination ethnique qui acquiert des dimensions inquiétantes (fig. I2). Malheureusement, Taste of Cement ne propose aucune contextualisation historico-politique de ce phénomène, ce qui aurait permis au public occidental peu informé de l'histoire du ProcheOrient de replacer le cas des ouvriers au cœur du récit dans le cadre plus large des relations entre la Syrie et le Liban, traversées - depuis leur fondation comme Etats souverains il y a près d'un siècle - par une tension constante. Toutefois, le discours symbolique du film, tel qu'il se cristallise dans ses dernières images, évite toute mise en accusation simpliste et univoque; l'idée de la réversibilité des positions et des rôles, véhiculée par ses plans ultimes, offre une représentation visuelle des rapports de force dynamiques et instables entre les deux peuples mis en regard.

En effet, lors de la guerre civile libanaise (I975-1990), la Syrie avait participé activement aux opérations militaires; qui plus est, à la fin de la guerre et pendant quinze ans (jusqu'en 2005), le Liban fut sous occupation syrienne. En 20II, six ans après le départ de l'armée syrienne du sol libanais, la guerre civile éclate en Syrie. A travers un effet de duplication de l'Histoire, ce fut cette fois le tour du Liban de se retrouver pris
17 Par ailleurs, il semble que les autorités libanaises ont mis en place un fichage systématique de la population syrienne, permettant - entre autres - l'interdiction d'accès des réfugiés à certains métiers. En outre, dans certaines régions, comme celle de la petite communauté de Hadeth, près de Beyrouth, des dizaines de commerces syriens ont été fermés ou confiés à des entrepreneurs libanais sous décision des instances politiques locales. 
18 Voir Elizabeth Picard, Liban Syrie, intimes étrangers. Un siècle d'interactions sociopolitiques, Arles, Actes Sud, coll. Sindbad, 20ı6. Selon Picard, politologue et spécialiste du Moyen Orient, à la fin de la guerre civile libanaise, l'armée syrienne est entrée au Liban - sur ordre du président de la République syrienne, Hafez el-Assad, père du président actuel, Bachar el-Assad - par crainte d'une révolution palestinienne qui emporterait le Liban et qui causerait, inévitablement, une intervention israélienne à l'issue imprévisible pour toute la région. Ajoutons, encore, le fait que la distribution de Taste of Cement intervient juste une année après le centième anniversaire des accords Sykes-Picot qui furent à l'origine de la création de la Syrie et du Liban en tant que deux entités séparées. D’ailleurs, une prolifération de publications sur les relations entre les deux pays a accompagné cet anniversaire; voir, parmi d'autres, James Barr, Une Ligne dans le sable. Le conflit franco-britannique qui façonna le Moyen-Orient, Paris, Perrin/ Ministère de la Défense, 2017 [20II].

19 Le moteur de recherche Google propose une vaste série d'articles et de vidéos en réponse à la demande «que se passe-t-il vraiment en Syrie?». La plupart d'entre eux arborent cette question (ou d'autres similaires) comme titre, à l'instar d'une page facebook, créée en 20I3, censée diffuser la "vérité sur la Syrie»: www.facebook.com/pg/Verite. sur.la.Syrie/about/?ref=page_internal [consulté le 6 juin 20I8].

20 Ziad Kalthoum, «La caméra est une arme redoutable», Ballast, 3 janvier 2018 , en ligne: www.revue-ballast.fr/ziadkalthoum-camera-arme-redoutable/ [consulté le 2r mai 20I8]. Il ajoute un peu

dans les affrontements, face à une guerre qui a très vite débordé sur son territoire ${ }^{18}$. Ainsi, les bâtisseurs du film, comme d'ailleurs tous les réfugiés syriens qui deviennent la cible d'actes de racisme au Liban, ne sont que les victimes d'une Histoire tumultueuse et violente, d'un cercle vicieux entamé de longue date; les derniers plans filmés depuis l'intérieur de la bétonnière inscrivent symboliquement le destin de ces hommes et, par extension, celui des peuples syrien et libanais dans les courbes concentriques d'une spirale infernale.

Or, le manque de repères directionnels, stables et fixes qui caractérise ces mêmes plans communique également un sentiment de confusion face à la conjoncture historique qui lie aujourd'hui les deux pays. De cette manière, le film de Kalthoum participe d'un aveu général d'incompréhension et d'impuissance dans l'analyse de la situation actuelle en Syrie, relayé par l'ensemble des médias dominants, ce qui ne manque pas d'affaiblir toute velléité de protestation et de mobilisation de la part du public ${ }^{19}$. Il peut paraître alors surprenant que Kalthoum déplore fréquemment dans ses déclarations publiques, à l’instar de ses commentaires devant le public du Cinéma Oblò, l'inefficacité prétendue du cinéma à éveiller la conscience politique du spectateur et à le pousser à l'action: «Je ne suis pas sûr que l'art puisse changer quoi que ce soit, aujourd'hui. Les gens regardent un film et oublient ensuite la situation, une fois rentrés chez eux» ${ }^{20}$. Or, une question s'impose: quelle est la responsabilité des cinéastes eux-mêmes vis-à-vis de cette (absence de) réaction de la part du public? A quel point les ambitions artistiques de Taste of Cement - et leurs corollaires, à savoir l'accumulation de prix aux festivals et la reconnaissance internationale pour le réalisateur et ses collaborateurs - prennent-elles le dessus sur son potentiel à développer un véritable discours de contre-pouvoir? Le spectateur sort secoué de la projection, bouleversé par la violence des images et la cruauté des situations dépeintes, mais sa volonté d'engagement politique ne peut-elle que se heurter à un manque flagrant de cible (militer pour qui? contre qui?).

La fonction assignée à la voix over constitue, à notre sens, un indicateur probant des limites de la visée politique du film. Précisons d'em-

plus loin: «Pour changer les choses, il faut construire un pont entre le réalisateur et le public, pousser le public à pen- ser à ce qui se passe autour de lui, éveiller les consciences.» 
blée que, tout au long du récit, cette voix constitue le seul moyen de transmission d'informations verbales. En planant sur les images, elle révèle la présence d'une instance énonciative autrement invisible que «le spectateur est incité à associer à l'origine même de l'ensemble du discours audiovisuel ${ }^{21}$, selon une convention mise en place très tôt dans l'histoire du cinéma parlant. Dans le cas de Taste of Cement, cette convention n'est pas dénuée de fondement: c'est la voix du réalisateur, Ziad Kalthoum, qui énonce ce commentaire over, amalgamant sur un mode lyrique l'expérience réelle des bâtisseurs avec les souvenirs autobiographiques du cinéaste. Nonobstant les enjeux éthiques que soulève ce type de témoignage par procuration, Kalthoum assume son choix de substituer sa parole à celle des ouvriers :

«La première fois que j’ai posé ma caméra face à eux, ils sont venus me dire qu'ils n'avaient pas le droit de parler: ni de leur vie en Syrie, ni de ceux qui avaient détruit leurs maisons, ni de la guerre - par peur du régime. Ils n'ont pas l'autorisation non plus de parler de leurs conditions de vie et de travail difficiles, sinon déplorables, dans cet immeuble libanais - par peur du propriétaire des lieux. [...] A partir du moment où ils m’ont dit qu'ils avaient interdiction de s'exprimer, je me suis interrogé: pourquoi questionner des personnes qui n'ont pas le droit de parler et, surtout, ont peur de le faire? [...] Il est plus intéressant d'exprimer la manière qui est la mienne de considérer ce sujet, en tant que réalisateur, par le prisme de mon expérience et de mon point de vue.» 22

On a souvent critiqué le cinéma militant pour son emploi d'un commentaire over trop didactique, voire même démagogique. Cependant, il nous semble que Taste of Cement tombe dans le piège inverse: la voix over se contente ici d'offrir une psychologisation de la situation des ouvriers - plutôt banale, d'ailleurs, dans son ton poétique - évitant la moindre tentative d'analyse historico-politique; de la sorte, l'origine $\mathrm{du}$ «mal» reste détachée de tout contexte explicite et le film reproduit ce qu'il prétend dénoncer, à savoir la domination par l'interdiction de la liberté d'expression. De la sorte, la fonction potentielle du cinéma en tant que dispositif de contre-pouvoir se trouve neutralisée. Par ailleurs, à l'instar des bâtisseurs, condamnés au silence, le public cible du film - à l'«Occident» comme à l'«Orient» - se trouve régulièrement assailli par des informations systématiquement partielles qui provoquent et entretiennent la confusion autour de la situation actuelle en Syrie. Il devrait
21 Alain Boillat, «Des personnages érigés en narrateurs: les voix over chez Joseph L. Mankiewicz. Pour une approche énonciative du récit filmique», Cahiers de narratologie. Analyse et théorie narratives, $\mathrm{n}^{\circ} 20$, juillet 20II, p. 2 ; en ligne: https:// journals.openedition.org/narratologie/ 6295 [consulté le ro juin 20ı8]. A la douzième minute du film, la subordination de l'ensemble des matières d'expression filmique au narrateur over devient explicite: alors que ce dernier énonce le mot «fixe» («still»), tous les ouvriers dans le champ s'immobilisent instantanément et la bande sonore devient, pendant quelques minutes, complètement silencieuse. Du reste, cette séquence rend manifeste le rapport de force qui relie l'énonciateur aux ouvriers filmés: la caméra a beau cadrer de manière très serrée les visages de ces derniers, la distance qui nous sépare de leur réalité demeure irréductible.

22 Ziad Kalthoum, op. cit. 
23 Au sein d'une presse francophone globalement fort élogieuse, signalons le positionnement minoritaire de Nicolas Didier dans Télérama pour qui «[Kalthoum] reconduit maladroitement, avec ce documentaire, ce qu'il entend dénoncer: l'avalement du corps humain par le ciment et l'acier.», Télérama, 2 janvier 20ı8, en ligne: www.telerama.fr/ cinema/films/taste-of-cement,n5377243, critique.php [consulté le ıo juin 20ı8]. Or, on ne manquera pas de remarquer que le critique a, lui-même, recours à une formulation lyrique de ses objections, escamotant en fin du compte les informations qu'il faudrait traiter (qui vend le ciment, à qui profite l'acier, etc.). alors incomber au cinéma documentaire - qui se veut, de plus, politiquement engagé - de remédier à ces lacunes.

Au demeurant, Taste of Cement n'évite pas non plus l'écueil de l'esthétisation de la guerre: l'emploi inopportun du ralenti dans la séquence qui suit les efforts des sauveteurs tentant de retrouver des survivants suite à un raid aérien, alors que la caméra cadre frontalement des personnes piégées dans les ruines de leurs maisons, entraîne inévitablement la spectacularisation d'un drame bien réel. Ce choix stylistique pourrait se justifier par son inscription dans une stratégie de choc à visée politique; toutefois, ce serait ignorer que la réalité médiatique actuelle est saturée d'images violentes de la guerre en Syrie sans pour autant produire un quelconque effet mobilisateur auprès du public. Par conséquent, à un niveau à la fois rhétorique et esthétique, Taste of Cement ne se distingue guère de la masse infinie de reportages journalistiques qui se cantonnent à diffuser des scènes sensationnelles sans rien dévoiler sur les acteurs qui tirent les ficelles de cette industrie extrêmement lucrative qu'est la guerre, si ce n'est que par son esthétisme exacerbé. Si nous considérons le film comme une belle occasion ratée, c'est parce que son sujet initial nous semblait offrir à lui seul des clés permettant de pénétrer là où les médias dominants refusent d'aller: qui profite des travaux de reconstruction des grandes métropoles au Proche Orient? Selon quelles modalités les contrats de concession des travaux sont-ils attribués? Quelles sont les instances impliquées? Pour transformer effectivement sa caméra en arme politique - comme le dernier carton du Sergent immortel l'annonçait en mode programmatique - il aurait suffi à Kalthoum de poser les bonnes questions.

Exalter la beauté d'un film qui traite des thématiques du racisme et de la guerre n'est pas exactement faire son éloge ${ }^{23}$; sur ce point, il faudrait du reste préciser de quelle «beauté» on parle - selon quels codes et critères, imposés par qui, etc. A cet égard, une analyse du rôle des festivals dans l'instauration de normes contribuant à la fois à l'uniformisation stylistique de la production cinématographique mondiale ainsi qu’au nivellement de son discours idéologique, aurait été utile afin de s’interroger sur la rareté des films qui développent et diffusent des propos politiquement subversifs. 